\title{
A real time robust observer for an Agonist-Antagonist Variable Stiffness Actuator.
}

\author{
T. Ménard, G. Grioli and A. Bicchi
}

\begin{abstract}
We consider the problem of estimating the timevarying stiffness in real-time of a Variable Stiffness Actuator in an agonistic-antagonistic configuration. The estimation of the stiffness is done in two steps. First, we use operational calculus which provides a relation between the positions/velocities of the motors and the link, the torques of the motors and the stiffness. Second, we combine the obtained relation with a polynomial approximation of the stiffness and a recursive least square algorithm to fit the data. Simulations and experimental results are provided and demonstrate the effectiveness of the proposed approach.
\end{abstract}

\section{INTRODUCTION}

The flexibility in robot actuators has long been considered as a nuisance, because it lowers the accuracy of the control. In the past decade, an inverse trend started, due to the expansion of technology in everyday life, the physical Human Robot Interaction (phHRI) has become of importance. The idea of taking advantage of the compliance of the actuators has then been developed. Indeed, a compliant actuator allows to reduce the risk of injuries in case of an accidental impact between the robots and a human [1]. Furthermore, compliant actuators can be useful to preserve mechanism [2] or to save energy [3]. Such considerations have lead to the design of Variable Stiffness Actuator (VSA) [4].

More precisely, VSA are devices for which both the position and the stiffness of the system can be controlled. For a detailed study about the existing devices, see [5]. We consider here VSA which are made up of two actuators and a flexible transmission which presents nonlinear characteristics. For this category of VSA, two main approaches exist. First the motors are placed in an agonist-antagonist position and connected to the link via nonlinear springs: for example, this approach has been developed at the University of Pisa, where an experimental device has been built [6]. Another architecture for a VSA is an explicit stiffness variator, where a motor is dedicated to position control and the other one to stiffness control. The dynamics of the motors are decoupled. Such a device has

T. Ménard is with GREYC, University of Caen, 6 Bd du Maréchal Juin, 14050 Caen Cedex, France. E-mail: tomas.menard@unicaen.fr

G. Grioli and A. Bicchi are with Centro Interdipartimentale di Ricerca "E. Piaggio", University of Pisa, 56126, Italy and Italian Inst. of Technology, Advanced Robotics, 16163 Genova, Italy been developed e.g. at the Istituto Italiano di Tecnologia [7].

The problem of controlling both stiffness and position has been studied and some control law have been proposed [8], [9], [10]. While the first is open-loop, the latter are feedback control, then an accurate estimation of the stiffness is needed. The main issue is that the stiffness is not directly observable. It can be seen as a differential operator who described the variation of physical quantities. A common way to obtain an estimation of the stiffness is to deduce it from a mathematical model, but such a method is prone to error. Indeed, the mathematical model is usually complex and subject to high imprecision. Furthermore, the stiffness is intrinsically nonlinear for VSA.

For all these reasons, an on-line estimator for the stiffness is far preferable. Several stiffness-observer exist in the literature. A first approach to estimate the stiffness on-line has been proposed in [11], the proposed observer error estimation was shown in theory to be Uniformly Ultimately Bounded. This restriction has been alleviated in [12], where the observer is derived from a parametric approach coupled with an analytic differentiation. Both these methods focus on being the more general and less invasive, and are then based on the link side. Observers designed specifically for VSA devices have been proposed in [13] for AwAS device, combined with control in [14] for the VSA-II device and in [15] for an approach which only need position and velocity measurements. These approaches have several points in common. First they are based on the motor side. Second, they follow the same scheme: obtain an estimation of the flexibility torque of the flexible transmission, then, define a parametrization of the torque and finally perform an analytical differentiation of the obtained function. The drawback is that it is hard to ensure the convergence of this type of method because even if a sequence of functions converges (toward the flexibility torque), conditions under which the associated sequence of derivatives (the stiffness) converges are very restrictive and hard to prove in this case.

In this work, we propose a different technique for the estimation of variable stiffness for the Agonist-Antagonist VSA device, which tries to overcome some of the limitation of the previous approaches. Instead of trying to get an estimation of the torque of the flexible transmission, we 
first use operational calculus and differential algebra (see [16], [17] for theoretical foundations and [18], [19] for applications to control) in order to obtain a relation between the stiffness and the measured signals. A parametrization of the stiffness is further injected in this relation, and a Recursive Least Square allows to obtain an estimation of the stiffness.

The main advantage of this approach is that the relation obtained between the stiffness and the measured signals is exact and involves only integrals of these signals. Since we use only integrals of the measured signals, the proposed approach is very robust to noise. Furthermore, the convergence of this method is direct, since the relation obtained is exact, the convergence is only up to the persistent excitation of the signal.

The paper is organized as follow. Section II states the problem. In section III, the basics of operational calculus are recalled and the stiffness observer is derived. We compare the observer proposed here with existing approach in section IV. Section V report some results obtained applying the observer on an experimental data set. Finally, we conclude in section VI.

\section{Problem Statement}

In this paper, we consider an Agonist Antagonist Variable Stiffness Actuator (AA-VSA). Such a device consists of two motors connected in parallel to the driven link through nonlinear flexible transmissions (see figure 1). In this section, we recall briefly the dynamic model of an AA-VSA as in [6].

Let the positions of the two motors and the link be represented by $q_{1}, q_{2}$ and $q_{L}$, respectively. The variables $\tau_{1}$ and $\tau_{2}$ are the torques of the the motors and $\tau_{L}$ is the external torque on the link. The dynamics of the system are then given by the following equations:

$$
\begin{aligned}
\tau_{1}= & J_{1} \ddot{q}_{1}+b_{1} \dot{q}_{1}-f_{1}\left(q_{L}-q_{1}\right), \\
\tau_{2}= & J_{2} \ddot{q}_{2}+b_{2} \dot{q}_{2}-f_{2}\left(q_{L}-q_{2}\right), \\
\tau_{L}= & J_{L} \ddot{q}_{L}+b_{L} \dot{q}_{L}+g\left(q_{L}\right) \\
& +f_{1}\left(q_{L}-q_{1}\right)+f_{2}\left(q_{L}-q_{2}\right) .
\end{aligned}
$$

The first two equations (1)-(2) depict the dynamics of the motors, where $J_{1}, J_{2}$ are the moments of inertia and $b_{1}, b_{2}$ the dampings. The third equation (3) represents the dynamics of the link where $J_{L}$ is the inertia, $b_{L}$ the damping and $f_{1}\left(q_{L}-q_{1}\right)$ and $f_{2}\left(q_{L}-q_{2}\right)$ are the elastic torques of the variable stiffness coupling of each motor and the link. The remaining term $g\left(q_{L}\right)=m g \frac{l}{2} \cos \left(q_{L}\right)$ is the gravity term acting on the link of mass $m$ and length $l$. It is zero if the link moves in an horizontal plane.

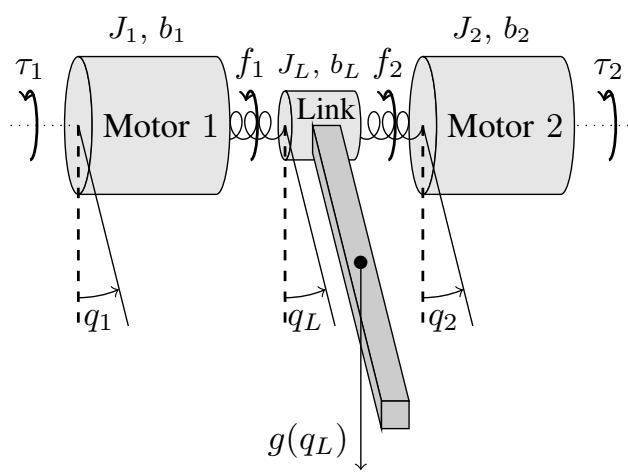

Fig. 1. Description of an Agonist-Antagonist VSA with definition of the variables.

The aim of this paper is to estimate the total device stiffness $\sigma$ which is associated to the total flexibility torque $f$ given by

$$
f(\phi)=f_{1}\left(\phi_{1}\right)+f_{2}\left(\phi_{2}\right)
$$

and

$$
\sigma(\phi)=\sigma_{1}\left(\phi_{1}\right)+\sigma_{2}\left(\phi_{2}\right)
$$

where

$$
\sigma_{i}\left(\phi_{i}\right)=\frac{\partial f_{i}}{\partial \phi_{i}}\left(\phi_{i}\right), \quad i=1,2
$$

and $\phi=\left(\phi_{1}, \phi_{2}\right)$ with $\phi_{i}=q_{L}-q_{i}, i=1,2$, correspond to the deformation of each motor-transmission.

\section{OBSERVER}

We work here from the motor side, that is, we use equations (1)-(2) to get an estimation of the stiffness. We assume to have access to $q_{1}, q_{2}, q_{L}$, the positions of the two motors and the link, and $\tau_{1}, \tau_{2}$ the torques of the two motors.

The method is divided into two steps. First, we use operational calculus framework in order to obtain a relation between the stiffness and the available signals. Then, we parametrize the stiffness by taking a Taylor expansion and inject it in the relation previously obtained. Finally, we apply a Recursive Least Square (RLS) algorithm to estimate the coefficients of the parametrization. We give hints for the application of the observer at the end of the section.

\section{A. Operational calculus framework}

We first recall the definitions and properties of operational calculus. Then, we apply it to our problem. 
Definitions and properties:

Operational calculus is based on the Laplace transform. Its main interest is that differential equations in the time domain correspond to algebraic equation in the operational domain.

Definition 1: [20] Let $f: \mathbb{R}_{+} \rightarrow \mathbb{R}$. If there exists $\beta \in \mathbb{R}$ such that the function $t \mapsto e^{-\beta t} f(t)$ is integrable on $\mathbb{R}_{+}$ then the Laplace transform of $f$ exists and is defined by:

$$
\hat{f}(s)=\mathcal{L}\{f\}(s)=\int_{0}^{+\infty} e^{-s t} f(t) d t,
$$

where $s \in \mathbb{C}$ and $\Re(s) \geq \beta$.

This transformation has numerous properties. We list hereafter the main properties which will be used here.

Proposition 1: [20] Let $n \in \mathbb{N}$ and $f: \mathbb{R}_{+} \rightarrow \mathbb{R}$ be a function such that its Laplace transform exists. The following properties hold true:

Derivative formula:

$$
\begin{gathered}
\mathcal{L}\left\{t^{n} f(t)\right\}(s)=(-1)^{n} \frac{d^{n}}{d s^{n}} \hat{f}(s) . \\
\mathcal{L}\left\{f^{(n)}(t)\right\}(s)=s^{n} \hat{f}(s)-s^{n-1} f(0)-\ldots-f^{(n-1)}(0) .
\end{gathered}
$$

Properties:

$$
\begin{gathered}
\mathcal{L}\left\{t^{n-1}\right\}(s)=\frac{(n-1) !}{s^{n}} . \\
\frac{1}{s^{n}} \hat{f}(s)=\mathcal{L}\left\{\frac{1}{(n-1) !} \int_{0}^{t}(t-\nu)^{n-1} f(\nu) d \nu\right\}(s) .
\end{gathered}
$$

Application to the VSA observer problem:

In order to derive a relation between the stiffness and the available signals, we start from equations (1)-(2):

$$
J_{i} \ddot{q}_{i}+b_{i} \dot{q}_{i}-f_{i}\left(q_{L}-q_{i}\right)=\tau_{i},
$$

where $i=1$ or 2 . We first take the derivative with respect to time of equation (12) in order to make the stiffness appear:

$$
J_{i} q_{i}^{(3)}+b_{i} \ddot{q}_{i}-\frac{\partial f_{i}}{\partial \phi_{i}}\left(\phi_{i}\right) \cdot \frac{d \phi_{i}}{d t}=\frac{d \tau_{i}}{d t} .
$$

Considering equation (13) in the operational domain and applying property (9), it becomes:

$$
\begin{aligned}
& s \hat{\tau}_{i}(s)-\tau_{i}(0)=J_{i}\left(s^{3} \hat{q}_{i}(s)-s^{2} q_{i}(0)-s \dot{q}_{i}(0)-\ddot{q}_{i}(0)\right) \\
&+b_{i}\left(s^{2} \hat{q}_{i}(s)-s q_{i}(0)-\dot{q}_{i}(0)\right) \\
&-\mathcal{L}\left\{\sigma_{i}\left(\phi_{i}\right) \frac{d \phi_{i}}{d t}\right\}(s) .
\end{aligned}
$$

In order to supress the dependance on the initial conditions, we take the third derivative with respect to $s$ of equation
(14):

$$
\begin{aligned}
& 3 \frac{d^{2}}{d s^{2}} \hat{\tau}_{i}(s)+ s \frac{d^{3}}{d s^{3}} \hat{\tau}_{i}(s)= \\
& J_{i}\left(6 \hat{q}_{i}(s)+18 s \frac{d}{d s} \hat{q}_{i}(s)+9 s^{2} \frac{d^{2}}{d s^{2}} \hat{q}_{i}(s)+s^{3} \frac{d^{3}}{d s^{3}} \hat{q}_{i}(s)\right) \\
&+b_{i}\left(6 \frac{d}{d s} \hat{q}_{i}(s)+6 s \frac{d^{2}}{d s^{2}} \hat{q}_{i}(s)+s^{2} \frac{d^{3}}{d s^{3}} \hat{q}_{i}(s)\right) \\
&+\frac{d^{3}}{d s^{3}} \mathcal{L}\left\{\sigma_{i}\left(\phi_{i}\right) \frac{d \phi_{i}}{d t}\right\}(s) .
\end{aligned}
$$

Roughly speaking, $s$ corresponds to the derivative in the time domain. Thus, in order to overcome the need of derivatives of the measured signals, we divide equation (15) by $s^{4}$. Going back into the time domain, we fix $t=T>0$ and apply properties (8) and (11), we then obtain:

$$
\begin{aligned}
& \int_{0}^{T} \frac{1}{6}(T-\nu)^{3} \nu^{3} \sigma_{i}\left(\phi_{i}(\nu)\right) \frac{d \phi_{i}}{d t}(\nu) d \nu= \\
& \int_{0}^{T} J_{i}\left((T-\nu)^{3}-9(T-\nu)^{2} \nu+9(T-\nu) \nu^{2}-\nu^{3}\right) q_{i}(\nu) d \nu \\
& \int_{0}^{T} b_{i}\left(-(T-\nu)^{3} \nu+3(T-\nu)^{2} \nu^{2}-(T-\nu) \nu^{3}\right) q_{i}(\nu) d \nu \\
& \left.\int_{0}^{T}-\frac{1}{2}(T-\nu)^{3} \nu^{2}+\frac{1}{2}(T-\nu)^{2} \nu^{3}\right) \tau_{i}(\nu) d \nu
\end{aligned}
$$

In order to obtain a more reactive relation, we take the integral over a finite time moving window. This is done by considering the signal Heaviside $(\varrho) y(\varrho+t)$ instead of $t \rightarrow y(t)$ in the previous equations. This simply means that we move the time origin from 0 to $\varrho$. We apply this and, in addition, we take $t=\varrho+T>0$ and we apply the change of coordinates $u=T \nu$ to have constant bounds on the integrals. Finally, we obtain:

$$
\begin{aligned}
T^{7} \int_{0}^{1} \frac{1}{6} w_{3,3}(u) \sigma_{i}( & \left.\phi_{i}(t+T(u-1))\right) \frac{d \phi_{i}}{d t}(t+T(u-1)) d u= \\
+ & T^{4} \int_{0}^{1} J_{i} \cdot G_{1}(u) q_{i}(t+T(u-1)) d u \\
+ & T^{5} \int_{0}^{1} b_{i} \cdot G_{2}(u) q_{i}(t+T(u-1)) d u \\
& +T^{6} \int_{0}^{1} G_{3}(u) \tau_{i}(t+T(u-1)) d u
\end{aligned}
$$

where

$$
\begin{aligned}
G_{1}(u) & =w_{3,0}(u)-9 w_{2,1}(u)+9 w_{1,2}(u)-w_{0,3}(u), \\
G_{2}(u) & =-w_{3,1}(u)+3 w_{2,2}(u)-w_{1,3}(u), \\
G_{3}(u) & =-\frac{1}{2} w_{3,2}(u)+\frac{1}{2} w_{2,3}(u),
\end{aligned}
$$

and $w_{\kappa, \mu}(u)=(1-u)^{\kappa} u^{\mu}$.

We can notice that we still need the first derivative of the displacement in the left-hand side of equation (17), it will be settled after the parametrization is done. The main advantage of the relation obtained in this section is that it is exact, indeed, there is no approximation made. 


\section{B. Parametrization and $R L S$}

We assume that both stiffness functions $\sigma_{i}, i=1,2$ can be written as a Taylor series expansion and we consider the truncature at order $k$ :

$$
\sigma_{i}\left(\phi_{i}\right) \approx \sum_{j=0}^{k} a_{j}^{i} \frac{\left(\phi_{i}\right)^{j}}{j !}
$$

where $a_{j}^{i}$ are constant parameters. We inject (18) into (17) which gives:

$$
C_{i}(t)=\sum_{j=0}^{k} a_{j}^{i} \cdot b_{j}^{i}(t)
$$

where

$$
\begin{aligned}
C_{i}(t)=\int_{0}^{1}\left(J_{i} \cdot G_{1}(u)\right. & \left.+T b_{i} \cdot G_{2}(u)\right) q_{i}(t+T(u-1)) d u \\
& +\int_{0}^{1}\left(T^{2} G_{3}(u)\right) \tau_{i}(t+T(u-1)) d u
\end{aligned}
$$

and

$$
\begin{aligned}
b_{j}^{i}(t) & \\
= & \frac{T^{3}}{6} \int_{0}^{1} \frac{w_{3,3}}{u}(t+T(1-u)) \cdot\left(\frac{\mathrm{d} \phi_{i}}{\mathrm{dt}} \times \frac{\left(\phi_{i}\right)^{j}}{j !}\right)(t+T(1-u)) d u, \\
= & -T^{2}\left[w_{3,3}(u) \cdot \frac{\left(\phi_{i}\right)^{j+1}}{(j+1) !}(t+T(1-u))\right]_{0}^{1} \\
& +\frac{T^{2}}{6} \int_{0}^{1} \frac{d w_{3,3}}{d u}(t+T(1-u)) \cdot \frac{\left(\phi_{i}\right)^{j+1}}{(j+1) !}(t+T(1-u)) d u, \\
= & \frac{T^{2}}{6} \int_{0}^{1} \frac{d w_{3,3}}{d u}(t+T(1-u)) \cdot \frac{\left(\phi_{i}\right)^{j+1}}{(j+1) !}(t+T(1-u)) d u .
\end{aligned}
$$

The second equality is obtained by applying an Integration by part. The first term in the right-hand side of the second equality is equal to zero because the function $w_{3,3}$ vanishes in 0 and 1 , which give the third equality.

In more compact form, we have:

$$
C_{i}(t)=A_{i}^{T} B_{i}(t),
$$

where $A_{i}$ and $B_{i}$ are column vectors such that the $j$-th coordinate is $a_{j}^{i}$ and $b_{j}^{i}$, respectively.

Finally, we apply the following RLS algorithm in order to get an estimate $\hat{A}_{i}$ of $A_{i}$ :

$$
\begin{aligned}
\dot{\hat{A}}_{i}(t) & =-P(t) B_{i}(t)\left(\hat{A}_{i}^{T} B_{i}(t)-C_{i}(t)\right), \\
\dot{P}(t) & =-P(t) B_{i}(t) B_{i}^{T}(t) P(t), \\
P(0) & =P(0)^{T}>0 \\
\hat{A}_{i}^{j}(0) & =0, \quad j=1, \ldots, k+1 .
\end{aligned}
$$

\section{Implementation of the algorithm}

We follow here the same lines as in [21]. Indeed, the coefficients of equation (19) are in the form

$$
\int_{0}^{1} g(u) y(t+T(1-u)) d u
$$

where $g$ is known and fixed before the experiment, while $y$ is measured during the experiment. We consider regularly spaced samples with a sampling period $T_{s}$. Let $y_{m}$ denote $y\left(m T_{s}\right)$. For an estimation time $T=M T s$, we set $t_{m}=m T_{s} / T$ and $g_{m}=g\left(m T_{s} / T\right)=g(m / M)$ for $m=0, \ldots, M$.

Let now $W_{m}$ be the weights corresponding to the trapezoidal method, that is, $W_{0}=W_{M}=T_{s} / 2$ and $W_{m}=T_{s}$, $m=1, \ldots, M-1$.

The integral is given numerically by:

$$
\int_{0}^{1} f(t) d t \approx \sum_{m=0}^{M} W_{m} f\left(t_{m}\right)
$$

which gives:

$$
\int_{0}^{1} g(u) y(t+T(1-u)) d \nu \approx \sum_{m=0}^{M} W_{m} g_{m} y_{l-m} .
$$

Finally, the coefficients are obtained from the output of a classical Finite Impulse Response (FIR) digital filter with impulse response $c_{m}=W_{m} g_{m}, m=0, \ldots, M$ (for more details about FIR filter, see [22]).

\section{Simulation Results}

In this section, the performance of the proposed observer is illustrated and compared with existing approach on simulations.

\section{A. Simulation}

We consider an Agonist-Antagonist VSA mechanism realized with two identical cubic springs whose force displacement characteristic is described by:

$$
f_{i}=10 .\left(q_{i}-q_{L}\right)^{3} .
$$

The parameters of the motors in the equations (1)-(2)-(3) are given by, $J_{1}=J_{2}=10^{-4} \mathrm{~kg} \cdot \mathrm{m}^{-2}, J_{L}=0.0179 \mathrm{~kg} \cdot \mathrm{m}^{-2}$, $b_{1}=b_{2}=1.27$ N.m.s $/$ Rad and $b_{L}=0.0127$ N.m.s $/$ Rad. We consider the case without gravity, that is $g=0$.

Two sinusoidal signal for $\tau_{1}$ and $\tau_{2}$ are given to the VSA device as input and shown on figure $2 b$ ). We present a simulation with a white noise on the measured data on figure 2 .

We compare here the approach proposed in this paper with the approach proposed in [15] which is based on a residual estimation of the flexibility torque.

set-up for the algorithm proposed in this paper

Since the force displacement is cubic, it is sufficient in equation (18) to take $k=2$. The parameters of the observer are set as follow, the length of the time window for the integral is $T=1 s$ and the covariance matrix for the RLS is initialized at $P(0)=10^{10} I d_{3}$. The sampling time is taken 
as $T s=1 / 1000$.

set-up for the approach proposed by Flacco et al. in [15] We just recall here the values of the different parameters needed. For more details, the interested reader is refered to [15] and [23]. The gain $K_{I}$ for the residual based flexibility torque estimation is set as $K_{I}=10^{3}$. The sampling time is the same for both approaches: $T s=1 / 1000$. The order of the basis for the least square estimation is $k=3$. The Recursive least square is started with $P(0)=10^{6} I d_{4}$ and the parameters to be estimated are initialized at zero. The derivative of the displacement angle is obtained with a Kinematic Kalman filter (see [24]) and set as in [23].

\section{Comparison of the two approaches}

We can notice on figure 2 that both approaches provide satisfying results. For a more precise comparison, we have computed the Mean Square Error (MSE) and the Mean Square Relative Error Percentage (MSREP):

$$
\begin{aligned}
\text { MSE } & =\frac{\sum_{k=0}^{p}[\sigma(k)-\hat{\sigma}(k)]^{2}}{p} \\
\text { MSREP } & =\frac{\sum_{k=0}^{p}\left[\left(\frac{\sigma(k)-\hat{\sigma}(k)}{\sigma(k)}\right)\right]^{2}}{p}
\end{aligned}
$$

after the convergence of the methods, that is between $2 s$ and $10 \mathrm{~s}$. The results is given in the following table:

\begin{tabular}{|c||c|c|}
\hline & Operational calculus & Flacco et al. \\
\hline \hline MSE & 4.2 & 51.8 \\
\hline MSREP & 0.7 & 22.26 \\
\hline
\end{tabular}

We see that the approach proposed here show much better performance. Another advantage of the approach proposed here is that it relies on an exact expression which does not involve any time derivative of the measured signals, then we don't need to compute numerical time derivative and the convergence of this method only depends on the persistent excitation of the regression vector given by equation (21) while the approach of Flacco et al. can hardly be proved since it relies on the fact that the derivatives sequence corresponding to a convergent sequence of functions converge as well.

\section{EXPERIMENTAL RESULTS}

\section{A. Set-up description}

The algorithm has been tested on an experimental VSA device with exponential springs. We do not recall here the characteristics of this device and the model based estimate, which are fully described in [11].

Due to uncertainties in the model of the actuator and in the identification of the model parameters, the knowledge of the true stiffness is reliable up to an error about $25 \%$ represented by the horizontal line in figure $3 \mathrm{~d}$ ). a) positions of the motors and the link

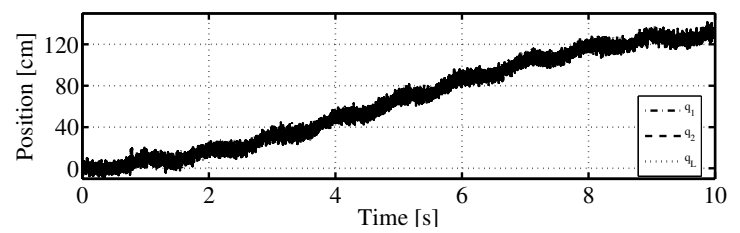

b) torques of the motors

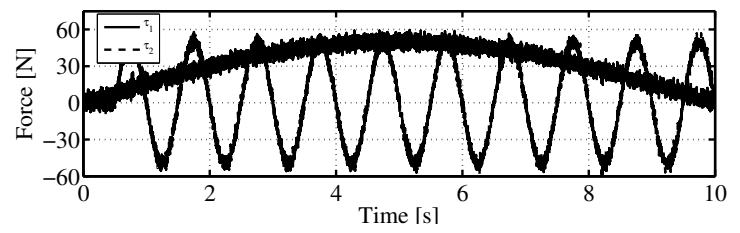

c) total stiffness and its approximation given by the approach proposed in this paper

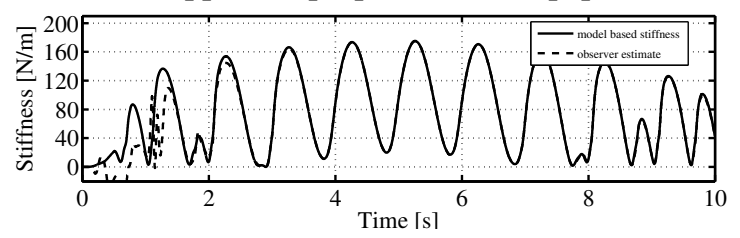

d) total stiffness and its approximation given by the approach proposed by flacco et al.

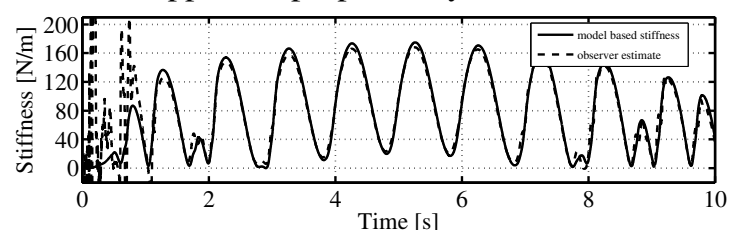

Fig. 2. Results of simulation: performance of the observer tracking the stiffness of an Agonist-Antagonist VSA system with white noise on the measured data.

The order of the series expansion has been set to 10 for both sides of the VSA device. The covariance matrix for the RLS (25) has been initialized with $P(0)=10^{7} I_{10}$.

\section{B. Results}

The data received from the device is reported on figures $3 a)$ and $3 b$ ). The stiffness is reconstructed in real-time as shown on figure $3 \mathrm{c}$ ) and the relative error between the modelbased and observer-based estimate is presented on figure $3 \mathrm{~d}$ ).

\section{CONCLUSION AND FUTURE WORK}

In this paper, we have presented an observer which reconstruct the stiffness of a Variable Stiffness Actuator in real-time using the positions of the motors and the link and the torques of the motors. Two points have been improved compared to the existing observer. First, no derivative of the measured signal is needed. Second, the convergence is ensured, provided that the signal given to the RLS is persistently excited. Comparison with the existing observer 
a) positions of the motors and the link

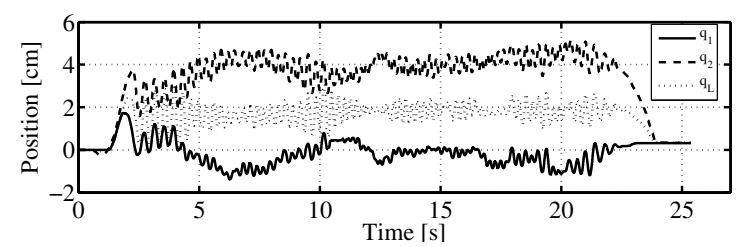

b) torques of the motors

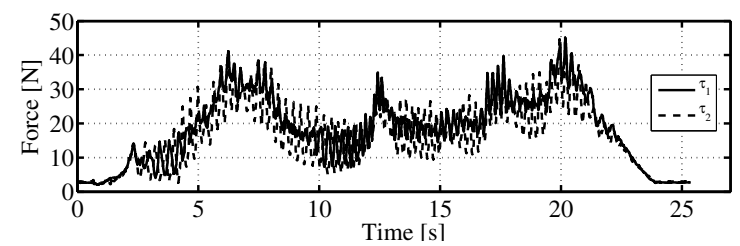

c) total stiffness and its approximation

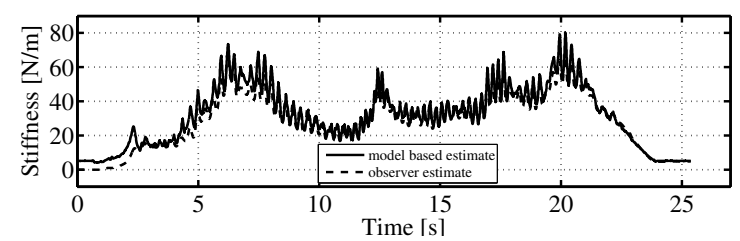

d) relative error

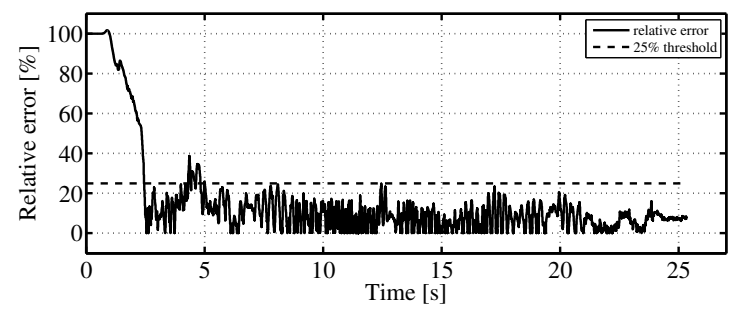

Fig. 3. Experimental results: data obtained from the AA-VSA and results obtained using the observer presented in this paper.

on simulation show that we obtain better performance. Furthermore, it is applicable in practice, as demonstrated by the experimental part.

The tunning of the observer and particularly the length of the time window for the integral has to follow a tradeoff between reactivity and robustness. A more detailed study for the set up of the observer will be adressed in future work.

\section{ACKNOWLEDGMENT}

This work was partially supported by the European Community, under FP7 grant ICT-287513 SAPHARI - Safe and Autonomous Physical Human Aware Robot Interaction.

\section{REFERENCES}

[1] A. Bicchi and G. Tonietti, "Fast and soft arm tactics: Dealing with the safety-performance trade-off in robots arms design and control," IEEE Robotics and Automation Magazine, vol. 11(2), pp. 22-33, 2004.
[2] S. Haddadin, T. Laue, U. Frese, S. Wolf, A. Albu-Schaeffer, and G. Hirzinger, "Kick it like a safe robot: requirements for 2050," in Proceedings of Robotics and Autonomous systems, 2009.

[3] M. Uemura and S. Kawamura, "Resonance-based motion control method for multi-joint robot through combining stiffness adaptation and iterative learning control," in Proceedings of Robotics and Automation, 2009.

[4] R. Van Ham, T. Sugar, B. Vanderborght, K. Hollander, and D. Lefeber, "Review of actuators with passive adjustable compliance/controllable stiffness for robotic apllications," IEEE Robotics and Automation Magazine, vol. 16(3), pp. 81-94, 2009.

[5] R. Ham, T. Sugar, B. Vanderborght, K. Hollander, and D. Lefeber, "Compliant actuator designs," Robotics Automation Magazine, IEEE, vol. 16, no. 3, pp. 81 -94, september 2009.

[6] R. Schiavi, G. Grioli, S. Sen, and A. Bicchi, "Vsa-ii: A novel prototype of variable stiffness actuator for safe and performing interacting with humans," in Proceedings of Robotics and Automation, 2008, pp. 21712176.

[7] A. Jafari, N. Tsagarakis, B. Vanderborght, and D. Cladwell, "A novel actuator with adjustable stiffness (awas)," in Proceedings of Intelligent Robots and Systems, 2010, pp. 4201-4206.

[8] G. Tonietti, R. Schiavi, and A. Bicchi, "Design and control of a variable stiffness actuator for safe and physical human/robot interaction," in Proccedings of Robotics and Automation, 2005, pp. 528-533.

[9] A. De Luca, F. Flacco, A. Bicchi, and R. Schiavi, "Nonlinear decoupled motion-stiffness control and collision detection/reaction for the vsa-ii variable stiffness device," in Proceedings of Intelligent Robots and Systems, 2009, pp. 5487-5494.

[10] A. De Luca and F. Flacco, "Dynamic gravity cancellation in robots with flexible transmissions," in Proceedings of Decison and Control, 2010, pp. 288-295.

[11] G. Grioli and A. Bicchi, "A non-invasive real-time method for measuring variable stiffness," in Robotics science and systems, Zaragoza, Spain, 2010.

[12] _ _ "A real-time parametric stiffness observer for vsa devices," in Proceedings of Robotics and Automation, 2011, pp. 5535-5540.

[13] F. Flacco, A. De Luca, I. Sardellitti, and N. Tsagarakis, "Robust estimation of variable stiffness in flexible joints," in Proceedings of Intelligent Robots and Systems, 2011, pp. 4026-4033.

[14] F. Flacco and A. De Luca, "Stiffness estimation and nonlinear control of robots with variable stiffness actuation," in Proceedings of IFAC World Congress, 2011, pp. 6872-6879.

[15] — - "Residual-based stiffness estimation in robots with flexible transmissions," in Proceedings of Robotics an Automation, 2011, pp. $5541-5547$.

[16] J. Mikusiǹski, Operational calculus, 2nd ed. PWN \& Pergamon, 1983, vol. 1.

[17] J. Mikusiǹski and T. Boehme, Operational calculus, 2nd ed. PWN \& Pergamon, 1987, vol. 2.

[18] M. Fliess and H. Sira-Ramírez, "An algebraic framework for linear identification," ESAIM COCV, vol. 9, pp. 151-168, 2003.

[19] M. Fliess, R. Marquez, E. Delaleau, and H. Sira-Ramírez, "Correcteurs proportionnels-intégraux généralisés," ESAIM COCV, vol. 7, pp. 2341, 2002.

[20] M. Abramowitz and I. Stegun, Handbook of mathematical functions, 1965.

[21] M. Mboup, C. Join, and M. Fliess, "Numerical differentiation with annihilators in noisy environment," Numerical algorithms, vol. 50(4), pp. 439-467, 2009.

[22] S. Haykin and B. Van Veen, Signals and Systems, 2nd ed. John Wiley \& Sons, 2002.

[23] F. Flacco, A. De Luca, I. Sardellitti, and N. Tsagarakis, "On-line estimation of variable stiffness in flexible robot joints," International Journal of Robotics Research, 2012.

[24] S. Jeon and M. Tomizuka, "Benefits of acceleration measurements in velocity estimation and motion control," IFAC Control Engineering Practice, vol. 15(3), pp. 325-332, 2007. 